\title{
Screening Test for At-Risk Drinking: Development of New Abbreviated Version of Alcohol Use Disorder Identification Test for Young and Middle-Aged Adults
}

\author{
Jae Hee Lee $\mathbb{D}^{1},{ }^{1}$ Koo Young Jung, ${ }^{2}$ and Yoon Hee Choi $\mathbb{D}^{2}$ \\ ${ }^{1}$ Department of Emergency Medicine, Ewha Womans University Mokdong Hospital, Seoul, Republic of Korea \\ ${ }^{2}$ Department of Emergency Medicine, College of Medicine, Ewha Womans University, Seoul, Republic of Korea \\ Correspondence should be addressed to Yoon Hee Choi; like-lemontea@hanmail.net
}

Received 5 February 2018; Revised 6 April 2018; Accepted 10 April 2018; Published 17 May 2018

Academic Editor: Chak W. Kam

Copyright (C) 2018 Jae Hee Lee et al. This is an open access article distributed under the Creative Commons Attribution License, which permits unrestricted use, distribution, and reproduction in any medium, provided the original work is properly cited.

Several abbreviated versions of the Alcohol Use Disorders Identification Test (AUDIT) have been developed for use in high-volume clinical situations such as emergency departments. In this study, we developed a new abbreviated version of AUDIT called the Screening Tool for At-risk Drinking (STAD) for young and middle-aged adults, consisting of two questions that reflect the structure of the AUDIT questionnaire using data from the Korea National Health and Nutrition Examination Survey (KNHANES). To derive the abbreviated test considering AUDIT item structure, we performed confirmatory factor analysis on the 10 AUDIT questions in the Korea National Health and Nutrition Examination Survey (KNHANES) IV. To validate the new abbreviated test, we analyzed the sensitivity, specificity, and area under the receiver operating characteristic curve (AUROC) on the KNHANES V-VI except for the KNHANES VI-2. Based on the two-factor structure of AUDIT, question (Q) 3 and Q7 were finally selected for STAD. In validation, AUROC was significantly wider for STAD than for AUDIT-QF, which has the same number of questions. There was no significant difference between AUDIT-C, consisting of three questions, and STAD. It can be used as a simple and reliable screening test in clinical settings.

\section{Introduction}

The Alcohol Use Disorder Identification Test (AUDIT) developed by the World Health Organization (WHO) in 1989 is now the most widely used test method to screen for atrisk drinking [1]. The development of AUDIT was based on multinational studies starting in 1987. The developers collected data consisting of 150 questions and sorted them into conceptual groups. Four groups were selected through correlation analysis and factor analysis of intrascale reliability and daily mean alcohol consumption. Then, the developers selected two or three questions per group, considering the weighted mean item-to-total correlation coefficient in each of the four groups; from these candidates, they assembled AUDIT, consisting of 10 questions [1-3]. AUDIT questions 1 to $3(\mathrm{Q} 1-\mathrm{Q} 3)$ are related to the amount and frequency of drinking, questions 4 to $6(\mathrm{Q} 4-\mathrm{Q} 6)$ are related to alcohol dependence symptoms, and questions 7 to 10 (Q7-Q10) are related to alcohol-related problem.

In United States, alcohol abuse is one of the main public health problems among young adults $[4,5]$. Despite the brief intervention, young adults still have alcohol drinking problems [6]. According to the data from Korea National Health and Nutrition Examination (KNHANES) conducted from 2007 to 2015, the risk drinking group was $41.2 \%$ in the $20 \mathrm{~s}, 35.5 \%$ in the $30 \mathrm{~s}, 33.4 \%$ in the $40 \mathrm{~s}$, and $29.0 \%$ in the $50 \mathrm{~s}$. However, it decreases in $60 \mathrm{~s}$ as $21.5 \%$, in the 70 s as $13.7 \%$, and in the $80 \mathrm{~s}$ and older as 5.9\% [7]. Screening and brief intervention for at-risk drinking among young and middleaged adults are important. National Institute on Alcohol Abuse and Alcoholism (NIAAA) proposed several clinical situations as key opportunities for screening at-risk drinking. It includes emergency department or urgent care center, prescribing a medicine that interacts with alcohol, and so on 
[8]. Many studies have shown that brief intervention about alcohol drinking in emergency department is effective [912]. Emergency department is an important clinical place for screening at-risk drinking and conducting brief intervention. However, it is not practically feasible to carry out all 10 AUDIT questions in many outpatient clinics or crowded emergency department. Therefore, research has been conducted to develop and test abbreviated versions of AUDIT as simple screening instruments that can be administered quickly [13-20].

For AUDIT-Q3 alone, AUDIT-QF, and AUDIT-C, items were selected based on past researches [19, 21], whereas AUDIT-4 and AUDIT-PC extracted the items using logistic regression $[14,22]$. FAST used a method to compare the sensitivity and specificity of the combinations of the questions after the questions were analyzed by principal component analysis [23]. However, previous abbreviated tests have a limitation in that they do not properly reflect the AUDIT survey structure. As the number of questions increases, the accuracy will increase. However, since it is not easy to conduct the tests, we tried to develop a two-question abbreviated test to replace AUDIT-C, which is widely used today.

At the time the AUDIT was developed, it consisted of three domains: Q1 to Q3, alcohol consumption factors; Q4 to Q6, alcohol dependence factors; and Q7 to Q10, harmful alcohol use. However principal component analysis and factor analysis in most studies supported a two-factor structure consisting of Q1-Q3 and Q4-Q10 [24-37]. This means that the structure of AUDIT in 3 domains can be divided into two major categories. In this study, we tried to develop new abbreviated test designated for young and middle-aged adults, the Screening Tool for At-risk Drinking (STAD), consisting of two questions that reflect the structure of the AUDIT questionnaire, using data from KNHANES, which are representative of the Republic of Korea.

\section{Material and Methods}

2.1. Setting and Data Collection. Data from the fourth KNHANES (KNHANES IV, 2007-2009) were used to extract questions for STAD. Data were obtained by requesting primitive data through the National Health and Nutrition Survey homepage of the Korea Centers for Disease Control and Prevention [38]. The sample design of the KNHANES IV used the 2005 Population and Housing Census data as a sampling framework. The first extraction unit was the village, the second extraction unit was the population housing survey unit, and the third extraction unit was the household. Socioeconomic location indexes and weights as well as a common variable survey, a health questionnaire, an examination survey, a nutrition survey, and other measures were conducted for selected subjects in the survey area. The health questionnaire contains 12 alcohol-related questions, including 10 questions based on the AUDIT 10 questions and two questions about drinking experience. For the validation of the newly developed abbreviated test, we used the alcohol-related contents of the fifth KNHANES (KNHANES V, 2010-2012) and the sixth KNHANES (KNHANES VI, 2013-2015). The alcohol-related contents were the same except in the second year of the KNHANES VI (KNHANES VI-2, 2014). Therefore, the data from the KNHANES VI-2 were excluded.

2.2. Outcome Measure. Among the subjects between 19 and 64 years old in the KNHANES IV, we applied the cutoff values (8 for males, 7 for females) for at-risk drinking proposed by $\mathrm{WHO}$ and divided the sample into normal and at-risk drinking groups [39]. To derive an abbreviated test considering the item structure of AUDIT, we performed confirmatory factor analysis on AUDIT 10 questions of the KNHANES IV. Based on the previous literature, we adopted a two-factor structure consisting of Q1-Q3 and Q4-Q10 [24-36]. In determining the number of questions in a new abbreviated test, the area under the receiver operating characteristic curve (AUROC) increases when the number of questions increases, but the function of the abbreviated test is inferior. Considering that AUDIT-C, the most widely used existing abbreviated test, is composed of 3 questions, this study attempts to develop a simpler two-question abbreviated test. Based on the score distribution and confirmatory factor analysis results of AUDIT 10 questions, one question was selected for each factor, and a new abbreviated test was derived. First, considering factor loading in the literature, we chose the questions based on the AUROC, sensitivity and specificity of each combination of questions. For the validation of the new abbreviated test, its sensitivity, specificity, and AUROC were analyzed on the KNHANES V-VI except for KNHANES VI-2.

2.3. Statistical Analysis. The fit of factor structure was assessed using chi-squared analysis, the Bentler comparative fit index (CFI), the root mean square error of approximation (RMSEA), and root mean square residual (RMSR). The cutoff values were $X^{2}, P>0.05$ [40]; CFI $>0.90$ [40]; RMSEA $\leq 0.08$ [41]; and RMSR $\leq 0.08$ [42]. For the validation of the new abbreviated test, the analysis was performed using the program SAS CALIS (SAS Institute, Cary, NC). The analysis programs PASW 18 and MedCalc Statistical Software version 17.2 (MedCalc Software bvba, Ostend, Belgium) were used.

\section{Results}

The KNHANES IV was conducted from 2007 to 2009 among 14,334 people aged between 19 and 64 years, including 6,278 men $(43.8 \%)$ and 8,056 women $(56.2 \%)$ with an average age of 42.3 years. Of these, $34.6 \%$ were in the at-risk drinking group (defined by a total AUDIT score of more than 8 for men, 7 for women). The KNHANES V VI except VI-2 was conducted from 2010 to 2013 and 2015 among 23,992 people aged between 19 and 64 years, including 10,450 men (43.6\%) and 13,542 women $(56.4 \%)$ with an average age of 43.5 years.

Based on our review of the previous literature on the factor structure of AUDIT, we adopted a two-factor structure consisting of Q1-Q3 and Q4-Q10. The chi-squared analysis and the CFI, RMSEA, and RMSR values that determine the fit of the factor structure were statistically significant. The items with the greatest factor loadings were Q3 in the first factor and Q7 and Q8 in the second factor (Table 1). After selecting Q3 from the first factor and Q7 and Q8 from the 
TABLE 1: Factor loadings for confirmatory factor analysis of AUDIT.

\begin{tabular}{lcccc}
\hline & Factor 1 & Factor 2 & Two-factor solution & Model fit \\
\hline Q1 & 0.78 & 0 & & \\
Q2 & 0.85 & 0 & & \\
Q3 & 0.96 & 0 & $X^{2}(\mathrm{df})$ & $1345.01(32), P<0.0001$ \\
Q4 & 0 & 0.76 & CFI & 0.9705 \\
Q5 & 0 & 0.73 & RMSEA & 0.07 \\
Q6 & 0 & 0.38 & RMSR & 0.03 \\
Q7 & 0 & 0.77 & & \\
Q8 & 0 & 0.77 & & \\
Q9 & 0 & 0.37 & 0.61 & \\
Q10 & 0 & & & \\
\hline
\end{tabular}

Notes. AUDIT $=$ Alcohol Use Disorders Identification Test CFI = comparative fit index; RMSEA = root mean square error of approximation; RMSR = root mean square residual.

TABLE 2: Comparison of AUROCs between Q3 + Q7 and Q3 + Q8 in KNHANES IV.

\begin{tabular}{lcccc}
\hline & Question combination & AUROC & $95 \%$ CI & $P$ value \\
\hline \multirow{2}{*}{ Male } & Q3 + Q7 & 0.97 & $0.964-0.973$ & $0.959-0.969$ \\
\multirow{4}{*}{ Female } & Q3 + Q8 & 0.964 & $0.973-0.980$ & $<0.0001^{*}$ \\
& Q3 + Q7 & 0.976 & $0.963-0.971$ & $<0.0001^{*}$ \\
\hline
\end{tabular}

Notes. AUROC $=$ area under the receiver operating characteristic curve; KNHANES IV $=$ the fourth Korea National Health and Nutrition Examination; CI $=$ confidence interval. $*$ means the significantly highest value than other groups.

second, we compared AUROC for the combinations of Q3, Q7 and Q3, Q8. The AUROC for the combination of Q3 and Q7 was greater; therefore, Q3 and Q7 were ultimately selected (Table 2).

The minimum total AUDIT score for the at-risk drinking group was defined as 8 for males and 7 for females. The cutoff values derived in the development of STAD were 3 for males and 2 for females. As a result, the sensitivity/specificity were 86.65/96.27 and 97.06/88.46, respectively, for male and female (Table 3). We compared the utility of the existing abbreviated tests AUDIT-QF and AUDIT-C with that of the new abbreviated test, STAD. AUROC was significantly wider for STAD than for AUDIT-QF, which has the same number of questions. There was no significant difference between AUDIT-C, consisting of three questions, and STAD (Table 4).

\section{Discussion}

AUDIT consists of 3 parts: questions 1 to 3 (Q1-Q3) are related to the amount and frequency of drinking, alcohol consumption; questions 4 to $6(\mathrm{Q} 4-\mathrm{Q} 6)$ are related to alcohol dependence symptoms; questions 7 to 10 (Q7-Q10) are related to alcohol-related problem. In the case of the existing abbreviated versions of AUDIT, the development was carried out in various ways, using the previous research $[19,21]$ and logistic regression analysis $[14,22]$ to select test items. In another abbreviated version of AUDIT, a principal component analysis was performed to select one question, and the remaining questions were combined to select the one with the highest sensitivity and specificity [23]. AUDIT-C, the most widely used abbreviated version of AUDIT [43], is limited to three questions about the consumption of alcohol and does not reflect the structure of the AUDIT 10 questions. As the number of questions increases, the accuracy of the abbreviated test will increase, but it becomes more difficult to conduct the abbreviated test. Therefore, we wanted to develop two-question abbreviated tests that could replace AUDIT-C, which is widely used at present. In clinical practice, many of the existing tests are abbreviated. For example, 12 items were selected among 36 items by regression analysis in the process of reducing the 36-Item Short-Form Health Survey (SF-36) to the 12-Item Short-Form Health Survey (SF-12) [44]. Another test, the CES-D scale (the Center for Epidemiologic Studies Depression Scale) for diagnosing depression, classifies many questions that have been verified as indicators of depression in previous studies as a set; 20 items were selected and classified under 4 factors [45]. Subsequently, 10 items that strongly correlated with the 4 factors in factor analysis were selected [46]. In this study, we used regression analysis to select two questions out of the 10 AUDIT questions; however, since the diagnostic criterion for at-risk drinking was the total score of the AUDIT 10 questions, no questions could be removed and the problem could not be addressed. Therefore, we decided to select the questions considering the factor structure of AUDIT. In the previous literature, test structures with one to three factors have been proposed. At the time when the AUDIT questions were developed, they consisted of three domains (Q1 to Q3, alcohol consumption factors; Q4 to Q6, alcohol dependence factors; and Q7 to Q10, harmful alcohol use factors). In the majority of studies, principal 
TABLE 3: Cut-off values, sensitivities, and specificities of STAD.

\begin{tabular}{|c|c|c|c|c|c|c|c|c|}
\hline & \multicolumn{2}{|c|}{ Sensitivity, \% } & \multicolumn{2}{|c|}{ Specificity, \% } & \multicolumn{2}{|c|}{$+\mathrm{LR}(95 \% \mathrm{CI})$} & \multicolumn{2}{|c|}{-LR (95\% CI) } \\
\hline \multicolumn{9}{|c|}{ STAD } \\
\hline \multicolumn{9}{|c|}{ Male } \\
\hline$\geq 2$ & 97.45 & $(96.8-98.0)$ & 77.24 & $(75.5-78.9)$ & 4.28 & $(4.0-4.6)$ & 0.033 & $(0.03-0.04)$ \\
\hline$\geq 3^{*}$ & 86.48 & $(85.3-87.6)$ & 95.63 & $(94.8-96.4)$ & 19.8 & $(16.5-23.8)$ & 0.14 & $(0.1-0.2)$ \\
\hline$\geq 4$ & 63.21 & $(61.5-64.9)$ & 99.56 & $(99.2-99.8)$ & 143.4 & $(79.5-258.8)$ & 0.37 & $(0.4-0.4)$ \\
\hline \multicolumn{9}{|c|}{ Female } \\
\hline$\geq 1$ & 99.42 & (98.9-99.7) & 70.16 & $(69.0-71.3)$ & 3.33 & $(3.2-3.5)$ & 0.008 & $(0.004-0.02)$ \\
\hline$\geq 2^{*}$ & 97.45 & $(96.5-98.2)$ & 88.01 & $(87.2-88.8)$ & 8.12 & $(7.6-8.7)$ & 0.029 & $(0.02-0.04)$ \\
\hline$\geq 3$ & 79.84 & $(77.6-81.9)$ & 98.07 & $(97.7-98.4)$ & 41.31 & $(34.6-49.4)$ & 0.21 & $(0.2-0.2)$ \\
\hline
\end{tabular}

Notes. STAD = screening test for at-risk drinking; LR = likelihood ratio; $\mathrm{CI}=$ confidence interval. $*$ means recommended cut-off values of STAD.

TABLE 4: Comparison of AUROCs between STAD and abbreviated version of the AUDIT in KNHANES V-VI.

\begin{tabular}{lcccrcr}
\hline & Question combination & AUROC & Test name & AUROC & $P$ value \\
\hline \multirow{2}{*}{ Male } & STAD (Q3 + Q7) & \multirow{2}{*}{0.97} & AUDIT-QF & 0.96 & 0.973 \\
& & & AUDIT-C & $0.0901^{*}$ \\
\multirow{2}{*}{ Female } & STAD (Q3 + Q7) & \multirow{2}{*}{0.976} & AUDIT-QF & 0.966 & $<0.0001^{*}$ \\
& & & AUDIT-C & 0.978 & 0.151 \\
\hline
\end{tabular}

Notes. AUROC $=$ area under the receiver operating characteristic curve; STAD $=$ screening test for at-risk drinking; AUDIT $=$ Alcohol Use Disorders Identification Test; KNANES V-VI $=$ the fifth and sixth Korea National Health and Nutrition Examination. * means the significantly highest value than other groups.

component analysis and factor analysis have supported a twofactor structure consisting of Q1-Q3 and Q4-Q10 [24-37]. In this study, we tried to construct a new abbreviated version of AUDIT with two questions by selecting one question from each of the two factors of AUDIT. For the first factor, we selected Q3, which had the highest factor loading, based on the results of the factor analysis and previous research [24-31]. For the second factor, Q7 and Q8, which had the highest factor loadings, were selected first. Subsequently, the combinations of Q3, Q7 and Q3, Q8 were compared in terms of AUROC, sensitivity, and specificity. Based on the results of the comparison, we selected Q3 and Q7 for the new abbreviated test, STAD. In the validation of the newly developed tests on the KNHANES V-VI except for the KNHANES VI-2, there is no statistically significant difference in AUROC between AUDIT-C and STAD. In other words, the number of questions was reduced, but the reliability of the test was unaffected. This means that STAD is superior in utility and addresses the structural limitations of AUDIT-QF and AUDIT-C, which are currently in widespread use.

This study uses the data of the KNHANES, the representative health indicator of the Republic of Korea, to develop a new abbreviated version of AUDIT based on the factor structure. We have presented effective means to screen for at-risk drinking in crowded environments such as emergency departments and primary care rooms. Drinking is not merely a subject of personal preference but a problem that needs to be assessed from a national perspective. The severe consequences of excessive alcohol consumption are already well known, and there is no question that systematic interventions should be made. It is important to understand the actual state of drinking in the Republic of Korea. Proper intervention must be applied to the at-risk population, and we facilitate such targeted intervention by developing a screening test for at-risk drinking. In addition, we believe our screening tool will facilitate research into the problem of alcohol abuse.

\section{Limitations}

The limitation of this study is that the questionnaire related to alcohol in the KNHANES did not record the exact amount of alcohol consumption or frequency of intake. If the criteria recommended by the National Institute on Alcohol Abuse and Alcoholism were used in the diagnosis of at-risk drinking, the study would be more reliable and easier to compare with other studies. Furthermore this study is based on data from public health research, it needed further studies to validate its usefulness in clinical settings.

\section{Conclusions}

This study developed efficient and accurate new abbreviated tests using national data. Specifically, we developed STAD, consisting of Q3, Q7 of AUDIT. We consider STAD to be a useful test reflecting the structure of AUDIT and the characteristics of each population group. It is expected that this test can replace previous abbreviated tests and facilitate screening for at-risk drinking.

\section{Data Availability}

The data used to support the findings of this study are available from the corresponding author upon request. 


\section{Conflicts of Interest}

The authors declare that there are no conflicts of interest regarding the publication of this paper.

\section{References}

[1] J. B. Saunders, O. G. Aasland, T. F. Babor, J. R. de la Fuente, and M. Grant, "Development of the alcohol use disorders identification test (AUDIT): WHO collaborative project on early detection of persons with harmful alcohol consumption-II," Addiction, vol. 88, no. 6, pp. 791-804, 1993.

[2] J. B. Saunders, O. G. Aasland, A. Amundsen, and M. Grant, "Alcohol consumption and related problems among primary health care patients: WHO Collaborative Project on Early Detection of Persons with Harmful Alcohol Consumption-I," Addiction, vol. 88, no. 3, pp. 349-362, 1993.

[3] J. B. Saunders, "The WHO Project on Early Detection and Treatment of Harmful Alcohol Consumption," Australian Drug and Alcohol Review, vol. 6, p. 6, 1987.

[4] J. M. Townshend, N. Kambouropoulos, A. Griffin, F. J. Hunt, and R. M. Milani, "Binge drinking, reflection impulsivity, and unplanned sexual behavior: impaired decision-making in young social drinkers," Alcoholism: Clinical and Experimental Research, vol. 38, no. 4, pp. 1143-1150, 2014.

[5] G. A. Bacio, V. M. Mays, and A. S. Lau, "Drinking initiation and problematic drinking among Latino adolescents: Explanations of the immigrant paradox," Psychology of Addictive Behaviors, vol. 27, no. 1, pp. 14-22, 2013.

[6] E. E. Tanner-Smith and M. W. Lipsey, "Brief alcohol interventions for adolescents and young adults: A systematic review and meta-analysis," Journal of Substance Abuse Treatment, vol. 51, pp. $1-18,2015$.

[7] Korea Centers for Disease Control and Prevention, "The Sixth Korea National Health and Nutrition Examination Survey (KNHANES VI)", 2015.

[8] M. L. Willenbring, S. H. Massey, and M. B. Gardner, "Helping patients who drink too much: an evidence-based guide for primary care clinicians," American Family Physician, vol. 80, no. 1, pp. 44-50, 2009.

[9] M. Van Loon, A. C. P. Van Gaalen, M. C. Van Der Linden, and C. Hagestein-De Bruijn, "Evaluation of screening and brief intervention for hazardous alcohol use integrated into clinical practice in an inner-city Emergency Department," European Journal of Emergency Medicine, vol. 24, no. 3, pp. 224-229, 2017.

[10] R. Patton and G. Green, "Alcohol identification and intervention in English emergency departments," Emergency Medicine Journal, vol. 35, no. 2, pp. 75-78, 2017.

[11] D. Duroy, I. Boutron, G. Baron, P. Ravaud, C. Estellat, and M. Lejoyeux, "Impact of a computer-assisted Screening, Brief Intervention and Referral to Treatment on reducing alcohol consumption among patients with hazardous drinking disorder in hospital emergency departments. The randomized BREVALCO trial," Drug and Alcohol Dependence, vol. 165, pp. 236-244, 2016.

[12] M. A. Walton, S. T. Chermack, F. C. Blow et al., "Components of brief alcohol interventions for youth in the emergency department," Substance Abuse, vol. 36, no. 3, pp. 339-349, 2015.

[13] K. A. Bradley, K. R. Bush, A. J. Epler et al., "Two brief alcoholscreening tests from the Alcohol Use Disorders Identification Test (AUDIT): Validation in a female Veterans Affairs patient population," Archives of Internal Medicine, vol. 163, no. 7, pp. 821-829, 2003.

[14] A. Gual, L. Segura, M. Contel, N. Heather, and J. Colom, "Audit3 and audit-4: Effectiveness of two short forms of the alcohol use disorders identification test," Alcohol and Alcoholism, vol. 37, no. 6, pp. 591-596, 2002.

[15] S.-I. Wu, H.-C. Huang, S.-I. Liu et al., "Validation and comparison of alcohol-screening instruments for identifying hazardous drinking in hospitalized patients in Taiwan," Alcohol and Alcoholism, vol. 43, no. 5, pp. 577-582, 2008.

[16] K. A. Bradley, A. F. Debenedetti, R. J. Volk, E. C. Williams, D. Frank, and D. R. Kivlahan, "AUDIT-C as a brief screen for alcohol misuse in primary care," Alcoholism: Clinical and Experimental Research, vol. 31, no. 7, pp. 1208-1217, 2007.

[17] D. A. Dawson, B. F. Grant, F. S. Stinson, and Y. Zhou, "Effectiveness of the derived Alcohol Use Disorders Identification Test (AUDIT-C) in screening for alcohol use disorders and risk drinking in the US general population," Alcoholism: Clinical and Experimental Research, vol. 29, no. 5, pp. 844-854, 2005.

[18] E. Burns, R. Gray, and L. A. Smith, "Brief screening questionnaires to identify problem drinking during pregnancy: A systematic review," Addiction, vol. 105, no. 4, pp. 601-614, 2010.

[19] K. Bush, D. R. Kivlahan, M. B. McDonell, S. D. Fihn, and K. A. Bradley, "The AUDIT alcohol consumption questions (AUDITC): an effective brief screening test for problem drinking. Ambulatory Care Quality Improvement Project (ACQUIP). Alcohol Use Disorders Identification Test," Archives of Internal Medicine, vol. 158, no. 16, pp. 1789-1795, 1998.

[20] J.-H. Seong, C.-H. Lee, H.-J. Do et al., "Performance of the AUDIT alcohol consumption questions (AUDIT-C) and AUDIT-K Question 3 alone in screening for problem drinking," Korean Journal of Family Medicine, vol. 30, no. 9, pp. 695-702, 2009.

[21] M. Aalto, M. Tuunanen, P. Sillanaukee, and K. Seppä, "Effectiveness of structured questionnaires for screening heavy drinking in middle-aged women," Alcoholism: Clinical and Experimental Research, vol. 30, no. 11, pp. 1884-1888, 2006.

[22] M. Piccinelli, E. Tessari, M. Bortolomasi et al., "Efficacy of the alcohol use disorders identification test as a screening tool for hazardous alcohol intake and related disorders in primary care: A validity study," British Medical Journal, vol. 314, no. 7078, pp. 420-424, 1997.

[23] R. Hodgson, T. Alwyn, B. John, B. Thom, and A. Smith, "The Fast Alcohol Screening Test," Alcohol and Alcoholism, vol. 37, no. 1, pp. 61-66, 2002.

[24] T. Chung, S. M. Colby, N. P. Barnett, and P. M. Monti, "Alcohol Use Disorders Identification Test: Factor Structure in an Adolescent Emergency Department Sample," Alcoholism: Clinical \& Experimental Research, vol. 26, no. 2, pp. 223-231, 2002.

[25] H. Bergman and H. Källmén, "Alcohol use among Swedes and a psychometric evaluation of the alcohol use disorders identification test," Alcohol and Alcoholism, vol. 37, no. 3, pp. 245-251, 2002.

[26] M. Shevlin and G. W. Smith, "The factor structure and concurrent validity of the alcohol use disorder identification test based on a nationally representative UK sample," Alcohol and Alcoholism, vol. 42, no. 6, pp. 582-587, 2007.

[27] S. R. Doyle, D. M. Donovan, and D. R. Kivlahan, "The factor structure of the Alcohol Use Disorders Identification Test (AUDIT)," Journal of Studies on Alcohol and Drugs, vol. 68, no. 3, pp. 474-479, 2007. 
[28] S. Cook, B. de Stavola, L. Saburova et al., "Socio-demographic predictors of dimensions of the audit score in a population sample of working-age men in Izhevsk, Russia," Alcohol and Alcoholism, vol. 46, no. 6, Article ID agr076, pp. 702-708, 2011.

[29] D. Wade, T. Varker, M. O’Donnell, and D. Forbes, “Examination of the latent factor structure of the Alcohol Use Disorders Identification Test in two independent trauma patient groups using confirmatory factor analysis," Journal of Substance Abuse Treatment, vol. 43, no. 1, pp. 123-128, 2012.

[30] T. Mathew, A. Shields, S. Yanov et al., "Performance of the alcohol use disorders identification test among tuberculosis patients in Russia," Substance Use \& Misuse, vol. 45, no. 4, pp. 598-612, 2010.

[31] M. Karno, E. Granholm, and A. Lin, "Factor structure of the Alcohol Use Disorders Identification Test (AUDIT) in a mental health clinic sample," Journal of Studies on Alcohol, vol. 61, no. 5, pp. 751-758, 2000.

[32] C. T. Lima, A. C. Freire, A. P. Silva, R. M. Teixeira, M. Farrell, and M. Prince, "Concurrent and construct validity of the audit in an urban brazilian sample," Alcohol and Alcoholism, vol. 40, no. 6, pp. 584-589, 2005.

[33] T. M. Kelly and J. E. Donovan, "Confirmatory factor analyses of the Alcohol use Disorders Identification Test (AUDIT) among adolescents treated in emergency departments," Journal of Studies on Alcohol, vol. 62, no. 6, pp. 838-842, 2001.

[34] B. von der Pahlen, P. Santtila, K. Witting et al., "Factor structure of the Alcohol Use Disorders Identification Test (AUDIT) for men and women in different age groups," Journal of Studies on Alcohol and Drugs, vol. 69, no. 4, pp. 616-621, 2008.

[35] G. Smith, M. Shevlin, J. Murphy, and J. Houston, "An assessment of the demographic and clinical correlates of the dimensions of alcohol use behaviour," Alcohol and Alcoholism, vol. 45, no. 6, pp. 563-572, 2010.

[36] C.-Z. Peng, R. W. Wilsnack, A. F. Kristjanson, P. Benson, and S. C. Wilsnack, "Gender differences in the factor structure of the Alcohol Use Disorders Identification Test in multinational general population surveys," Drug and Alcohol Dependence, vol. 124, no. 1-2, pp. 50-56, 2012.

[37] D. F. Reinert and J. P. Allen, "The alcohol use disorders identification test: an update of research findings," Alcoholism: Clinical and Experimental Research, vol. 31, no. 2, pp. 185-199, 2007.

[38] Centers for Disease Control and Prevention, "Nutrition Survey of KNHANES IV 2009”, 2009.

[39] T. Babor, J. C. Higgins-Biddle, J. B. Saunders, and M. G. Monteiro, AUDIT, The Alcohol Use Disorders Identification Test: Guidelines for Use in Primary Care, World Health Organization, Dept. of Mental Health and Substance Dependence, Geneva, Switzerland, 2001.

[40] P. M. Bentler, EQS Structural Equations Program Manual, BMDP Statistics Software, Los Angeles, Calif, USA, 1989.

[41] M. W. Browne and R. Cudeck, "Alternative ways of assessing model fit," Sociological Methods \& Research, vol. 21, no. 2, pp. 230-258, 1992.

[42] L. Hu and P. M. Bentler, "Cutoff criteria for fit indexes in covariance structure analysis: conventional criteria versus new alternatives," Structural Equation Modeling, vol. 6, no. 1, pp. 1$55,1999$.

[43] National Institute on Alcohol Abuse and Alcoholism, Helping patients who drink too much : a clinician's guide, U.S. Department of Health and Human Services, Rockville, MD, USA, 2007.
[44] J. E. Ware Jr., M. Kosinski, and S. D. Keller, "A 12-Item shortform health survey: construction of scales and preliminary tests of reliability and validity," Medical Care, vol. 34, no. 3, pp. 220233, 1996.

[45] L. S. Radloff, "The CES-D scale: a self-report depression scale for researching the general population," Applied of Psychological Measures, vol. 1, no. 3, pp. 385-401, 1977.

[46] J. G. Grzywacz, J. D. Hovey, L. D. Seligman, T. A. Arcury, and S. A. Quandt, "Evaluating short-form versions of the CES-D for measuring depressive symptoms among immigrants from Mexico," Hispanic Journal of Behavioral Sciences, vol. 28, no. 3, pp. 404-424, 2006. 


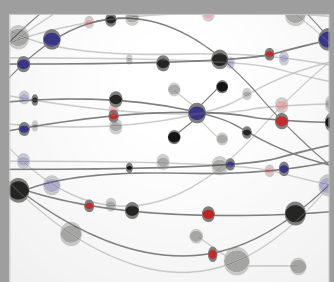

The Scientific World Journal
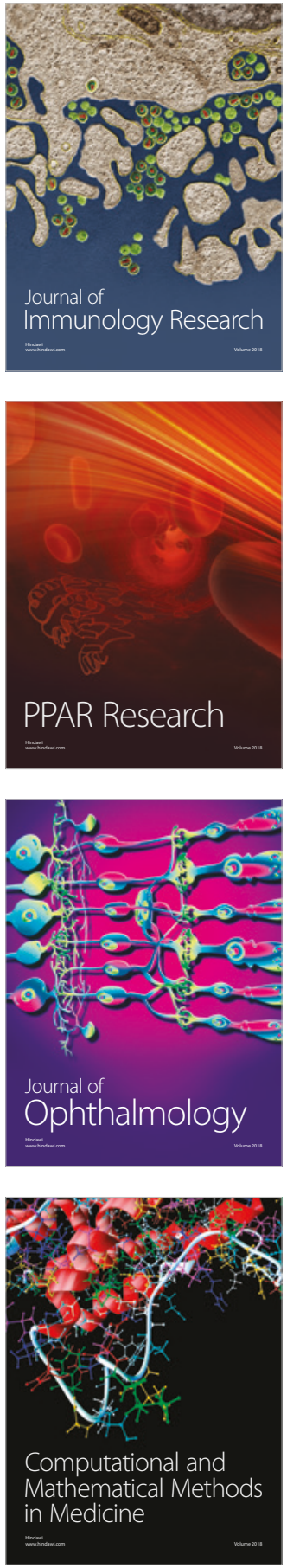

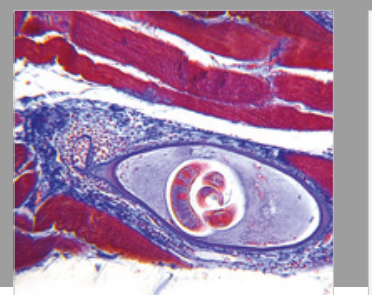

Gastroenterology Research and Practice

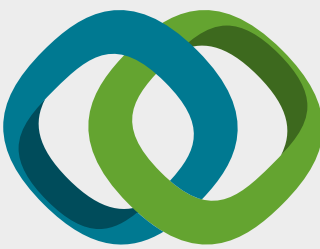

\section{Hindawi}

Submit your manuscripts at

www.hindawi.com
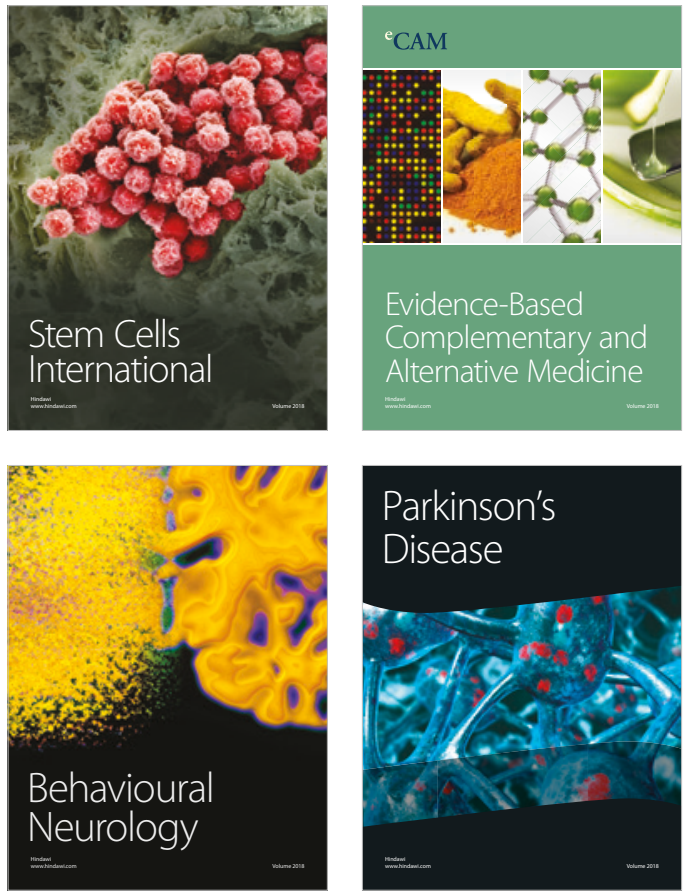

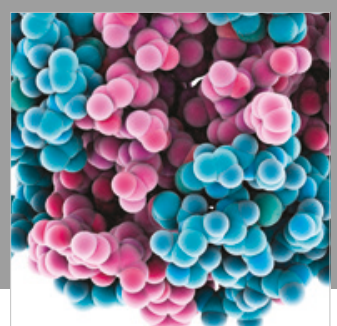

ournal of

Diabetes Research

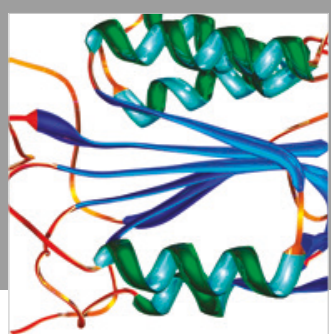

Disease Markers
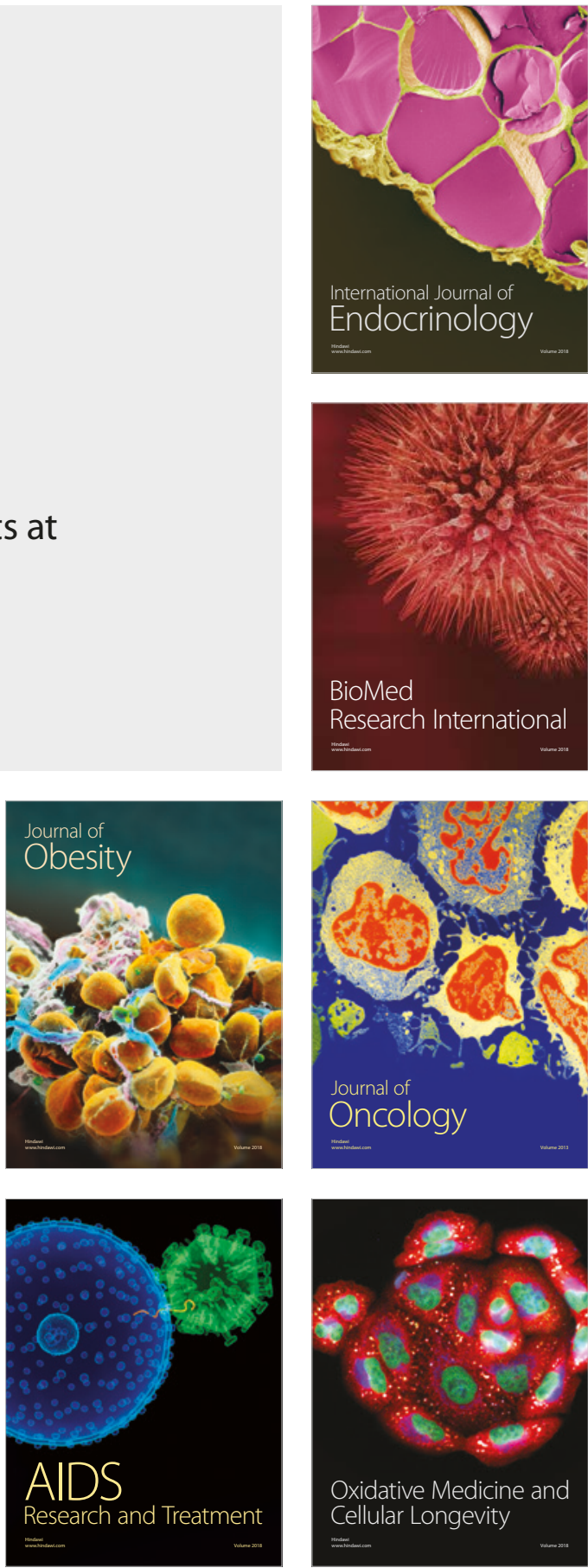